\title{
Laser printing of active optical microstructures
}

\author{
G. Koundourakis, C. Rockstuhl, D. Papazoglou, A. Klini, I. Zergioti, N. A. Vainos, ${ }^{a)}$ \\ and C. Fotakis ${ }^{\text {b) }}$ \\ Foundation for Research \& Technology_Hellas Institute of Electronic Structure and Laser, P.O. Box 1527, \\ Heraklion 71110, Greece
}

(Received 24 July 2000; accepted for publication 14 December 2000)

\begin{abstract}
Optically activated indium oxide diffractive optical microstructures are demonstrated through a two step laser based fabrication method. Nonstoichiometric indium oxide thin film material grown by reactive pulsed laser deposition has been selectively transferred onto glass substrates by ultrashort pulse laser microprinting to form surface relief microstructures. Subsequent ultraviolet illumination has induced dynamic refractive index changes, which result in all optical control of the grating diffraction efficiency. The direct materials transfer process enables reproduction of the structural and physical properties of the oxide. (C) 2001 American Institute of Physics.
\end{abstract}

[DOI: $10.1063 / 1.1348321]$

Indium oxide based thin-film compounds are currently finding several uses ${ }^{1,2}$ in display, image sensor, and solar cell technology, owing to their unique properties of high optical transparency and large controllable electrical conductivity. ${ }^{3,4}$ Their dynamic optical properties also address innovative applications in light modulation, switching, ${ }^{5}$ and holographic recording ${ }^{6}$ and merit further detailed fundamental and applied investigation.

Nonstoichiometric indium oxide $\left(\mathrm{InO}_{x}\right)$ and indium tin oxide (ITO) has been seen to exhibit large reversible electrical conductivity changes through cyclic photoreduction and oxidization processes, induced by UV light $\left(h \nu>E_{g}\right.$ $\sim 3.3 \mathrm{eV}-3.5 \mathrm{eV}$ ) illumination and exposure to ozone respectively. ${ }^{3,4,7}$ Electric current injection or ultraviolet illumination produces refractive index (and absorption) changes leading to optical modulation in optical waveguide configured polycrystalline ITO films. ${ }^{5}$ Efficient holographic recording in $\mathrm{InO}_{x}$ thin-films by low intensity $\mathrm{UV} \mathrm{HeCd}$ laser radiation $(325 \mathrm{~nm})$ has also been demonstrated. ${ }^{6,8}$ Oxygen vacancies play an important role in the aforementioned acting as donor centers enabling efficient excitation, charge transfer, and localized carrier concentration manipulation. ${ }^{3}$ These reversible effects result in relatively high localized refractive index changes of $\Delta n \sim 0.5 \%$ at the milliwatt power levels used.

Thin films grown by pulsed laser deposition (PLD) in an oxygen reactive atmosphere offer an enhanced and tunable holographic performance. ${ }^{8}$ This is attributed to the growth of a more efficient mesoscopic $\mathrm{InO}_{x}$ phase, which comprises micro/nanocrystals and metal clusters embedded in the amorphous matrix. The structure of the material and the experimental conditions are the main factors affecting the holographic UV light sensitivity. ${ }^{9}$ The crystallinity of the material which can be tuned during the PLD growth, influences the response time of the holographic recording. ${ }^{8}$ These effects, indicate that the rather complex electron transfer pro-

a) Author to whom correspondence should be addressed; present address: The National Hellenic Research Foundation, 48 Vas. Constantinou Ave., Athens 11635, Greece; electronic mail: vainos@eie.gr

${ }^{b)}$ Also at: University of Crete, Department of Physics, P.O. Box 2208, Heraklion 71003, Greece. cesses are favored at the crystallite interfaces, having an increased defect content. ${ }^{7}$ In addition, the use of ArF excimer laser radiation $(\lambda=193 \mathrm{~nm})$, below ablation or melting threshold conditions, produces permanent structural changes and fixed hologram recording. ${ }^{10,11}$

In a different context, laser induced spatially selective forward materials ablation and transfer ${ }^{12,13}$ have been applied for the direct production of optical microstructures. Ultrashort UV laser pulses have recently produced submicron structures ${ }^{14}$ even on highly curved substrates, thus opening up exciting microfabrication possibilities. ${ }^{15}$

Microprinting of transparent dielectrics was first attempted for the fabrication of computer generated $\mathrm{InO}_{x}$ surface relief holograms ${ }^{14}$ and the structure of the material has been verified by $x$-ray diffraction analysis. In this work, we further advance the transfer method and demonstrate experimentally that the directly grown surface-relief dielectric $\mathrm{InO}_{x}$ microstructures can be activated optically. By applying exclusively laser based methods, we produce, without any additional processing, a thin-film "target" material and transfer it selectively onto a "receiver" substrate preserving the dynamic optical properties of the material. The designed grating form of the microstructures enables the demonstration of optical activation effects by means of photoinduced diffraction efficiency changes.

We have fabricated $\mathrm{InO}_{x}$ thin-films on fused-silica plates by reactive PLD using pure indium metal and oxygen as outlined in Fig. 1(a). Experimental details and structural analysis of these films have already been presented in Ref. 7 . $\mathrm{InO}_{x}$ growth rates of $\sim 0.01-0.05 \mathrm{~nm} /$ pulse are achieved and films of thickness in the range of 100 to $400 \mathrm{~nm}$ exhibit excellent uniformity, high optical transparency in the visible and good holographic performance in the UV. These films have been subsequently used as the target materials in the laser microprinting ${ }^{14,15}$ operation outlined in Fig. 1(b). In some experiments, dc magnetron sputtered films have also been used. For the microprinting, a hybrid distributed feedback dye-laser/KrF excimer laser, delivering $\sim 500$ fs duration pulses of $\sim 10 \mathrm{~mJ} / \mathrm{pulse}$, at $\lambda=248 \mathrm{~nm}$, is used. The $\mathrm{InO}_{x}$ film is selectively ablated and redeposited on a Corning glass (UV grade) receiver substrate positioned nearby $(2-5$ 


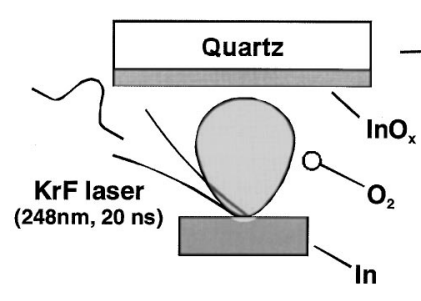

(a)

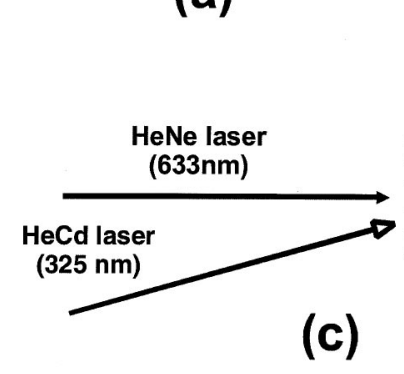

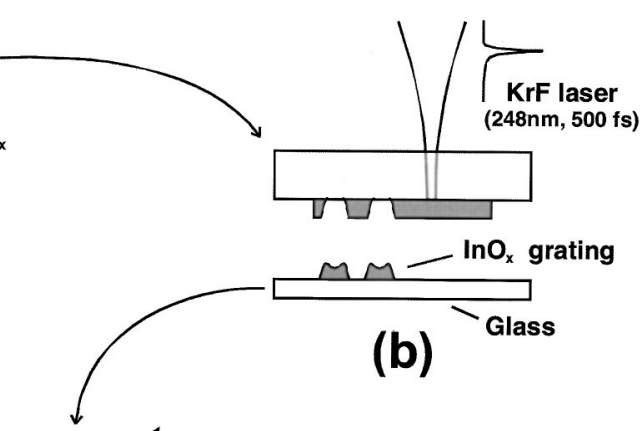

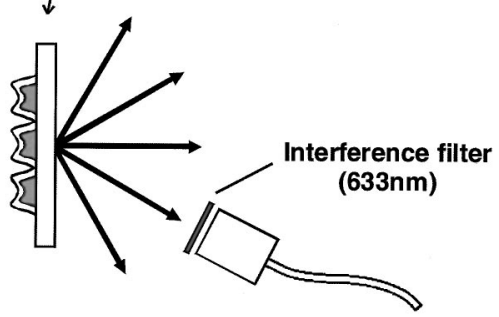

FIG. 1. Outline of $\operatorname{InO}_{x}$ grating development and testing (a) pulsed laser deposition of the $\mathrm{InO}_{x}$ targets, (b) $\mathrm{InO}_{x}$ grating microprinting, and (c) optical diffraction and grating activation. $\mu \mathrm{m}$ distance) in a low vacuum $\left(\sim 10^{-1}\right.$ Torr $)$ chamber. The substrate-source distance is monitored interferometrically. Relatively long slit features are projected demagnified by $\times 30$ to produce deposits of lateral dimensions $\sim 5 \mu \mathrm{m}$ $\times 100 \mu \mathrm{m}$. The maximum laser energy density was 400 $\mathrm{mJ} / \mathrm{cm}^{2}$ on target and a high-precision step-and-repeat operation was applied ${ }^{14}$ to form simple grating microstructures. The proper surface relief height was achieved by using a repetitive microprinting operation. Additionally, a thin $(10-20 \mathrm{~nm}) \mathrm{InO}_{x}$ overcoat was also applied by PLD and appears to optimize the activation effects.

All grating structures are transparent and exhibit permanent diffraction effects as evidenced by the monitoring $\mathrm{HeNe}$ laser probe beam as shown in Figure 1(c). The structures were analyzed with $\mathrm{x}$-ray diffractometry and the results were in full agreement with our previous work. ${ }^{14}$ The refractive index, $n$, and the extinction coefficient, $k$, of the oxide target materials have also been estimated by thin-film matrix methods at $n=2.0 \pm 0.03$ and $k=0.005 \pm 0.002$, using independent measurements at $\lambda=633 \mathrm{~nm}$. The larger error of $k$ is attributed to surface contaminations. Thus, the grating is expected to be mainly of a transmissive rather than of an absorptive nature. By producing a feature height of $316 \mathrm{~nm}$, the $\pi$-phase spatial wave front modulation $\left(n_{\mathrm{InO}_{x}} \approx 2.0\right.$ at $\lambda$ $=633 \mathrm{~nm}$ ) would allow optimum diffraction performance. Nevertheless, such an exact tuning was not pursued here. The smaller $(\sim 150-250 \mathrm{~nm})$ feature height grown results in lower diffraction efficiency $(<10 \%$ in each of the \pm 1 orders), which enables the determination of the sign of the refractive index changes discussed as follows. Grating spatial dimensions were restricted to the range of $2 \mathrm{~mm} \times 2 \mathrm{~mm}$ to allow for a full laser beam overlap.

To demonstrate grating activation, $\mathrm{HeCd}$ laser radiation is incident on the structure. Diffraction efficiency is monitored using a HeNe laser. Both the HeNe laser $(12 \mathrm{~mW}$ power at $633 \mathrm{~nm}$ ) and the $\mathrm{HeCd}$ laser $(38 \mathrm{~mW}$ at $325 \mathrm{~nm})$ are incident nearly normal to the substrate surface either unfocused or loosely focused. A fast shutter was also used in the UV beam to alternate between the ON and OFF activation states. Electrical activation of the structures has also been observed, but this will be discussed elsewhere. An atomic Downloaded 08 Dec 2008 to 194.177.215.121. Redistribution subject force microscope (AFM) image of part of a typical $\mathrm{InO}_{x}$ grating is shown in Fig. 2. Fourier analysis of its profile reveals a dominant period of $\sim 10 \mu \mathrm{m}$ that is in agreement with this grating design. Typical feature height values fall also within the design range of 150-200 $\mathrm{nm}$. The dual peak structure apparent on each grating line is formed during the deposition process, and is probably due to the high velocity of the ejected material or reablation effects. Subwavelength inhomogenities are inherent at the high laser intensities used. Their formation depends on the physical properties of the material and experimental conditions, although the mechanism is not known at this stage.

Optical activation experiments have been performed in the temperature range of $10{ }^{\circ} \mathrm{C}-55^{\circ} \mathrm{C}$ using a stabilized Peltier element. The \pm 1 -diffracted order beam intensity was recorded for consecutive activation cycles and the absence of fatigue effects has been verified. The $\mathrm{ON}$ and $\mathrm{OFF}$ states were established for long time periods to ensure relaxation of all processes involved. For unperturbed gratings of $\sim 180 \mathrm{~nm}$ nominal average relief height, the net (uncorrected for loss) background (dark) diffraction efficiency values are typically $\eta_{ \pm 1} \approx 5 \%$ and remain constant in time to about $\pm 0.1 \%$. Within the temperature range examined an overall $\pm 2 \%$ variation of diffraction efficiency of the grating has been observed owing to refractive index changes due to thermal excitation. Neither dependence on light polarization, nor any

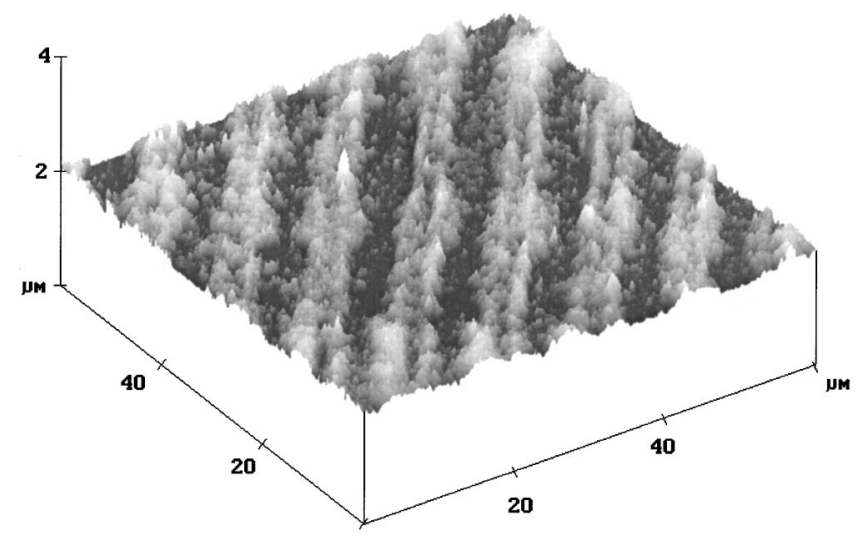

FIG. 2. AFM image of a typical $\mathrm{InO}_{x}$ microprinted grating on glass. AIP license or copyright; see http://apl.aip.org/apl/copyright.jsp 


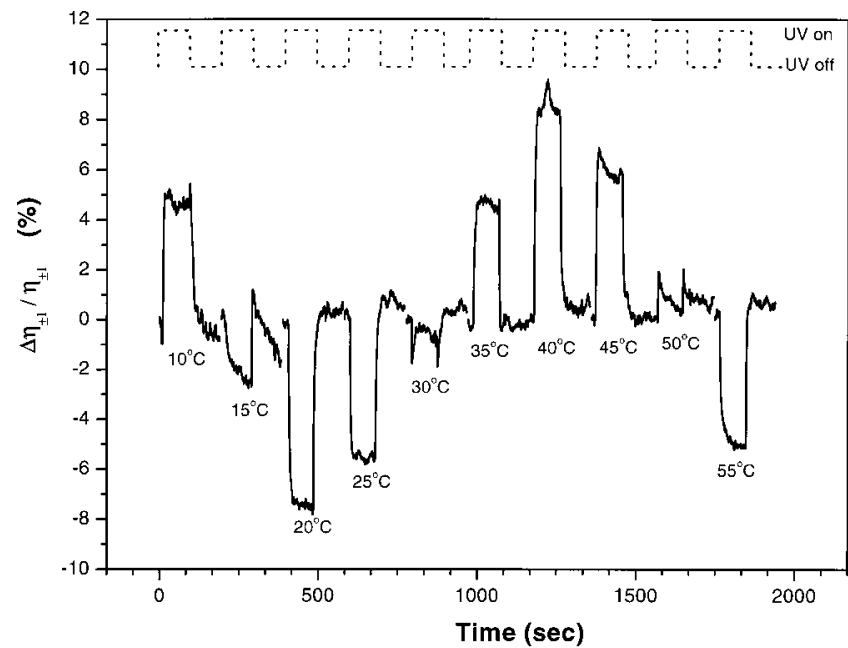

FIG. 3. Temporal evolution of relative change of the diffraction efficiency $\left(\Delta \eta_{ \pm 1} / \eta_{ \pm 1}\right)$ at various temperatures for activation UV intensity 1.9 $\mathrm{W} / \mathrm{cm}^{2}$.

effects due to the HeNe laser beam were observed. Higher diffraction orders exhibited a similar dynamic behavior.

The typical temporal evolution of the relative change in the diffraction efficiency $\left(\Delta \eta_{ \pm 1} / \eta_{ \pm 1}\right)$ of the structure upon UV illumination at various temperatures is depicted in Fig. 3. At a specific temperature the $\mathrm{ON}$ state produces a rise or a fall of the background (zero level) diffraction efficiency according to the respective refractive index changes induced. At the $633 \mathrm{~nm}$ probe wavelength $(h \nu \sim 1.9 \mathrm{eV}), k$ is minimal and at least two orders of magnitude lower than $n$. Absorption changes are thus expected to play a minor role. The ON state is established with typical time constant $\tau_{\mathrm{ON}} \sim 4 \mathrm{~s}$ in the temperature regions of $20^{\circ} \mathrm{C}$ and $45^{\circ} \mathrm{C}$ where maximum $\Delta \eta_{ \pm 1} / \eta_{ \pm 1}$ was recorded. The return to the original OFF state occurs at a comparable value of $\tau_{\mathrm{OFF}} \sim 4 \mathrm{~s}$ for these temperature values. Rigorous coupled wave analysis ${ }^{16}$ provides an estimate of $\Delta n \sim \pm 0.047$ (or $\sim 2.35 \%$ ) for the maximum induced refractive index changes. The suboptimal height of the relief assists in determining the sign of such changes. In our case, the optical thickness is less than that required to reach the first maximum in the diffraction curve, hence $\operatorname{sign}\left(\Delta \eta_{ \pm 1}\right)=\operatorname{sign}(\Delta n)$.

Examination of Fig. 3 reveals the presence of at least two competing processes. These processes, of opposite sign, are seen to be of comparable strength at temperatures, of $\sim 15^{\circ} \mathrm{C}, \sim 30^{\circ} \mathrm{C}$, and $\sim 50{ }^{\circ} \mathrm{C}$ and result in minimum diffraction efficiency $\Delta \eta_{ \pm 1}$ changes. $\Delta \eta_{ \pm 1} / \eta_{ \pm 1}$ is plotted in Fig. 4 for various activation intensity levels at three characteristic temperatures. The temperature of $30^{\circ} \mathrm{C}$ represents a local point of inflection.

According to Drude's model the electron photoreduced excitation is the basis for the decrease of the refractive index. On the other hand, oxidation in atmosphere and internal charge transfer between the active centers may justify the observed competing processes. These processes are strongly temperature dependent and their simultaneous action leads to refractive index sign reversal.

In conclusion, optically activated $\mathrm{InO}_{x}$ surface relief microstructures have been developed through laser based meth-

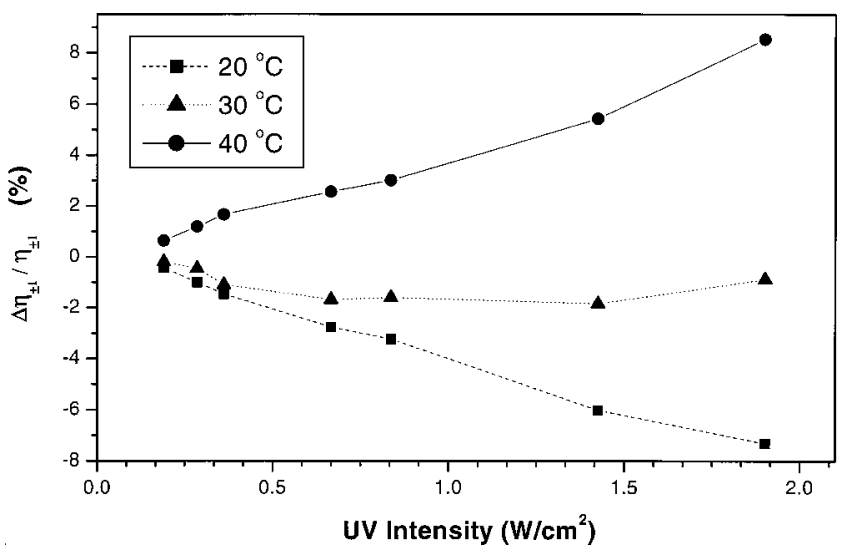

FIG. 4. Relative change of the diffraction efficiency $\left(\Delta \eta_{ \pm 1} / \eta_{ \pm 1}\right)$ for different intensities at three representative temperatures.

ods. Optical activation of these structures has been demonstrated by using near UV laser irradiation. The overall behavior shows close similarities with the previously reported holographic recording on $\mathrm{InO}_{x}$ thin films. The origin of the effects is not fully understood and further study is under way. The use of laser-based methods, in the fabrication of optically activated micro and nanostructures, opens up new and exciting application possibilities in the area of the optoelectronics.

This work was supported through European Union funded TMR-Ultraviolet Laser Facility, INCO-Inpulsenet and GROWTH-Holauthentic projects. Useful discussions and support of G. Kiriakidis, and A Perrone and D. Sakellaridis, are gratefully acknowledged.

${ }^{1}$ C. G. Granqvist, Appl. Phys. A: Mater. Sci. Process. 57, 19 (1993).

${ }^{2}$ I. Hamburg and C. G. Granqvist, J. Appl. Phys. 60, R123 (1986).

${ }^{3}$ B. Pashmakov, B. Claflin, and H. Fritzsche, J. Non-Cryst. Solids 164, 441 (1993).

${ }^{4}$ B. Claflin and H. Fritzsche, J. Electron. Mater. 25, 1772 (1996).

${ }^{5}$ R. T. Chen and D. Robinson, Appl. Phys. Lett. 60, 1541 (1992).

${ }^{6}$ S. Mailis, L. Boutsikaris, N. A. Vainos, C. Xiroulaki, G. Vasiliou, N. Garawal, G. Kiriakidis, and H. Fritzsche, Appl. Phys. Lett. 69, 2459 (1996).

${ }^{7}$ C. Xirouhaki, G. Kiriakidis, T. F. Pederson, and H. Fritsche, J. Appl. Phys. 79, 1 (1996).

${ }^{8}$ C. Grivas, D. S. Gill, S. Mailis, L. Boutsikaris, and N. A. Vainos, Appl. Phys. A: Mater. Sci. Process. 66, 201 (1998).

${ }^{9}$ K. Moschovis, E. Gagaoudakis, E. Chatzitheodoridis, G. Kiriakidis, S. Mailis, E. Tzamali, N. A. Vainos, and H. Fritzsche, Appl. Phys. A: Mater. Sci. Process. 66, 651 (1998).

${ }^{10}$ S. Pissadakis, S. Mailis, L. Reekie, J. S. Wilkinson, R. W. Eason, N. A. Vainos, K. Moschovis, and G. Kiriakidis, Appl. Phys. A: Mater. Sci. Process. 69, 333 (1999).

${ }^{11}$ T. Szorenyl, L. D. Laude, I. Bertoti, Z. Kantor, and Zs. Geretovsky, J. Appl. Phys. 78, 6211 (1995).

${ }^{12}$ J. Bohandy, B. F. Kim, and F. J. Adrian, J. Appl. Phys. 60, 1538 (1986).

${ }^{13}$ E. Fogarassy, C. Fuchs, F. Kerherve, S. Hauchecorne, and J. Perriere, J. Appl. Phys. 66, 457 (1989).

${ }^{14}$ I. Zergioti, S. Mailis, N. A. Vainos, P. Papakonstantinou, C. Kalpouzos, C. P. Grigoropoulos, and C. Fotakis, Appl. Phys. A: Mater. Sci. Process. 66, 579 (1998).

${ }^{15}$ S. Mailis, I. Zergioti, G. Koundourakis, A. Ikiades, A. Patentalaki, P. Papakonstantinou, N. A. Vainos, and C. Fotakis, Appl. Opt. 38, 2301 (1999).

${ }^{16}$ M. G. Moharam, Drew A. Pommet, and T. K. Gaylord, J. Opt. Soc. Am. A 12, 1077 (1995). 